\title{
Digital Sensing and Molecular Computation by an Enzyme-Free DNA Circuit
}

\author{
William E. Arter ${ }^{1,2}$, Yuriy Yusim ${ }^{1}$, Quentin Peter $^{1}$, Christopher G. Taylor ${ }^{1}$, David Klenerman ${ }^{1}$, Ulrich \\ F. Keyser ${ }^{2, \dagger} \&$ Tuomas P. J. Knowles ${ }^{1,2, \ddagger}$ \\ ${ }^{1}$ Department of Chemistry, University of Cambridge, Lensfield Road, Cambridge, CB2 1EW, UK \\ ${ }^{2}$ Cavendish Laboratory, University of Cambridge, JJ Thomson Avenue, Cambridge, CB3 OHE, UK \\ Corresponding authors: $\uparrow$ ufk20@cam.ac.uk, $\$$ tpjk2@cam.ac.uk
}

\begin{abstract}
DNA circuits form the basis of programmable molecular systems capable of signal transduction and algorithmic computation. Some classes of molecular programmes, such as catalysed hairpin assembly, enable isothermal, enzyme-free signal amplification. However, current detection limits in DNA amplification circuits are modest, as sensitivity is inhibited by signal leakage resulting from noncatalysed background reactions inherent to the non-covalent system. Here, we overcome this challenge by optimising catalysed hairpin assembly for single-molecule sensing in a digital droplet assay. Furthermore, we demonstrate digital reporting of DNA computation at the single- molecule level by employing ddCHA as a signal transducer for simple DNA logic gates. By facilitating signal transduction of molecular computation at $\mathrm{pM}$ concentration, our approach can improve processing density by a factor of $10^{4}$ relative to conventional DNA logic gates. More broadly, we believe that digital molecular computing will broaden the scope and efficacy of isothermal amplification circuits within DNA computing, biosensing and signal amplification in general.
\end{abstract}

\section{Keywords}

DNA nanotechnology, isothermal DNA circuitry, DNA computing, microfluidics, digital sensing, single molecule 
Signal amplification is an essential mechanism for efficient and reliable communication in biology. ${ }^{1}$ Biochemical cascades control cellular behaviour by mediating the molecular response to stimuli, by controlling the extent of signal amplification via a network of catalytic amplifiers. ${ }^{2,3}$ Transduction mechanisms can be highly sensitive, with molecular recognition of even a single initiator capable of producing a significantly amplified response., ${ }^{4,5}$

Inspired by biological signalling pathways, advances in DNA-based molecular programming have paved the way for artificial molecular catalysts capable of amplifying stimuli from nucleic acids, proteins or small molecules, that act as programmable intermediates between input and output signals. This has driven the development of several families of synthetic DNA signal amplifiers, which have found application in biological assays. ${ }^{6-10}$ These DNA circuits operate isothermally and without the need for extrinsic enzyme reagents, allowing facile integration into broader isothermal circuits built solely of DNA. ${ }^{11}$

Catalysed hairpin assembly (CHA), ${ }^{12}$ hybridisation chain reaction (HCR) ${ }^{13}$ and entropy-driven catalysis $(\mathrm{EDC})^{14}$ are the most prevalent examples of isothermal signal amplification circuitry. A common feature of these circuits are metastable DNA complexes that undergo transformation by strand displacement to a lower-energy conformation when triggered by an oligonucleotide catalyst. In the CHA reaction, for example, two complementary DNA hairpins are prevented from hybridisation by inhibition of the complementary sequences in the intramolecular self-binding region. A DNA strand of the correct base sequence can catalyse hairpin hybridisation by opening one hairpin through toehold-binding and branch migration, exposing a sequence domain complementary to the loop region of the other hairpin. This initiates hybridisation of the two hairpins through a further branch migration event, and results in release of the catalytic DNA by strand displacement for use in further catalytic cycles. The hybridised hairpins possess a newly-exposed domain, which produces a fluorescent, electrochemical or colorimetric signalling response by hybridisation with an appropriate molecular beacon. As is essential for sensing applications, signal amplification is highly specific, with precise base sequences in catalyst and circuit required for effective operation.

However, DNA amplification circuits are yet to achieve single-molecule sensitivity in signal transduction, as is observed in biological systems. This is a consequence of circuit leakage, a major obstacle to the application of DNA circuits, where background signalling occurs via reactions that 'short' the circuit in the absence of the analyte catalyst. Defective and subsequently misfolded DNA strands, the primary source of leak reactions, ${ }^{15,16}$ prevent limits of detection (LOD) beyond tens of pM even in highly-optimised circuits. ${ }^{17}$ Here, we negate this issue by employing digital microfluidics to incorporate a CHA circuit into a single molecule assay. The CHA reaction is well-suited to this purpose, as it is initiated in a 'one-pot' process and no further purification of commercially-available reagents is required. CHA reactions are compartmentalised within femtolitre-sized microreactors, so that single strands of encapsulated catalyst DNA have sufficient concentration to amplify a fluorescence signal above that of droplets not containing an analyte strand. Thus, an 'analogue' number of analyte molecules is binarised into droplets that produce a positive (1) or negative (0) signal. ${ }^{5,18}$ Using this method, we achieve detection sensitivity in the CHA reaction as low as $10 \mathrm{fM}$, by determining absolute analyte concentration according to the frequency of positive droplets. ${ }^{19}$

Furthermore, we employ our digital droplet CHA (ddCHA) platform to demonstrate the feasibility of digital, single-molecule chemical computing. Signal amplification circuits form a subset of the broader field of DNA-based molecular programming. ${ }^{20-22}$ By exploiting the simple rules that govern nucleotide 
base pairing, DNA nanosystems have been designed to perform logic operations ${ }^{23-25}$ and transduce molecular signals in vivo. ${ }^{26-29}$ Through manipulation of the ddCHA catalyst, we construct logic gates capable of fundamental Boolean operations, and demonstrate ddCHA signal transduction from these simple computational operations at $\mathrm{pM}$ signal concentra- tion. This represents a $10^{4}$ increase in signalling density compared to conventional computational circuits, ${ }^{30,31}$ where gate concentrations are typically on the order of tens of $\mathrm{nM}$. Therefore, our findings demonstrate the potential for ddCHA to facilitate significant improvements in circuit parallelisation and computational power in molecular programming.

\section{Results}

\section{Selection of DNA circuit for digital droplet assay}

We began by selecting and optimising the reaction conditions of a previously well-characterised CHA reaction for adoption within the digital assay. ${ }^{17}$ We consider the CHA reaction to be the most suitable DNA circuit for digital sensing in comparison to HCR and EDC; conventional HCR assays are unsuitable due to the necessary reagent addition and washing steps. FRET-based HCR approaches do not require washing steps, but the autocatalytic nature of the reaction means that any circuit leakage results in a strong false-positive signal. EDC reactions provide amplification in a 'one-pot' reaction, though typically at slower turnover rates compared to $\mathrm{CHA}^{7}{ }^{7}$ and the starting duplexes require laborious gel-based purification to ensure correct stoichiometry. Indeed, we set the constraint that all DNA reagents used should be commercially available, without the need for further in-house purification or non-trivial DNA syntheses, which otherwise limit the scalability of DNA circuitry.

The CHA reaction mechanism consists of a series of toehold-binding and strand displacement events by which a DNA catalyst enables hybridisation of otherwise metastable DNA hairpin motifs (Figure 1(a)). First, catalyst $\mathrm{C} 1$ binds to the exposed toehold of hairpin $\mathrm{H} 1$ before opening it through strand invasion (i), the newly exposed domains of $\mathrm{H} 1$ are then free to bind to the toehold region of and hybridise with $\mathrm{H} 2$, which ejects $\mathrm{C} 1$ through strand displacement in the process (ii). The released $\mathrm{C} 1$ strand is then free to catalyse another cycle, whilst the exposed region of the $\mathrm{H} 1: \mathrm{H} 2$ duplex goes on to hybridise with and displace the RQ quencher strand from the reporter duplex RFQ. This produces a turnon of fluorescence from the now unquenched fluorophore of RF in the final H1:H2:RF complex (iii). To establish the feasibility of ddCHA, we begin by optimising the reaction to function at the previouslyreported sensitivity limit of $15 \mathrm{pM} \mathrm{C1}$ catalyst. We then introduce the more powerful C5 amplifier (possessing $5 \times$ repeat units of $\mathrm{Cl}$ catalytic sequence, Figure 1(a)) to ensure sufficient signal strength for the assay to be robust to experimental noise. 
a
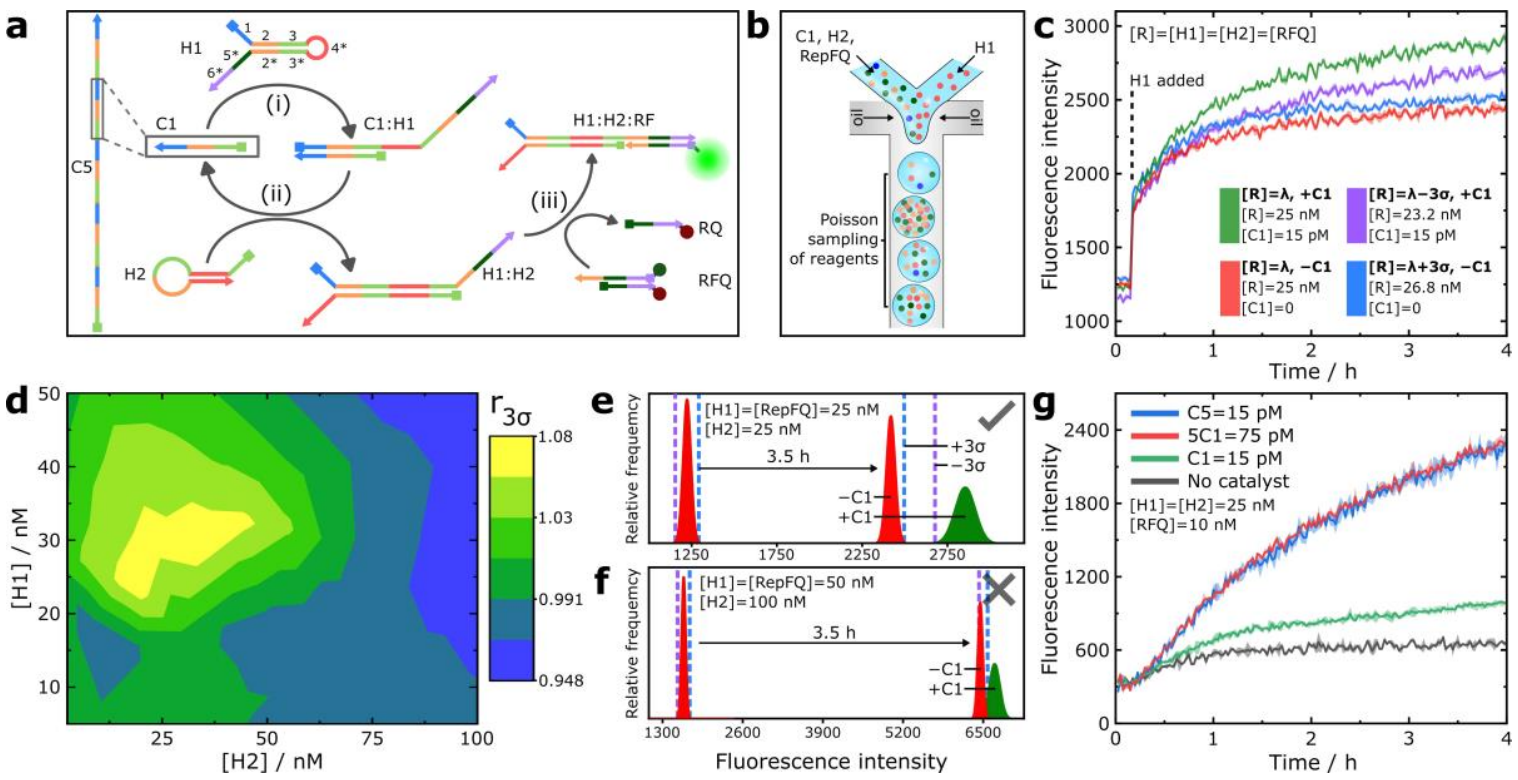

Figure 1: Optimisation of CHA reaction conditions. (a) Component oligonucleotides and scheme of CHA reaction. (i) $\mathrm{C} 1$ opens $\mathrm{H} 1$ by toehold binding and strand invasion, (ii) $\mathrm{C} 1$ is displaced by hybridisation of $\mathrm{H} 2$ to $\mathrm{H} 1$ through toehold binding to newly exposed $3^{*}$ domain of $\mathrm{H} 1$, (iii) displacement of quencher strand RQ from fluorescent reporter strand RF by the H1:H2 duplex. Sequence domains are labelled and colour coded, with complementary domains indicated by (*). (b) Cartoon depicting Poisson statistics of oligonucleotide encapsulation during droplet emulsification of the reaction mixture. (c) Real-time signal amplification (+C1) in bulk-phase CHA reaction for the $\lambda$ and $\lambda_{-3 \sigma}$ reagent concentration and background leakage reaction $(-\mathrm{C} 1)$ for the $\lambda$ and $\lambda_{+3 \sigma}$ reagent concentration corresponding to a $6 \mu \mathrm{m}$ droplet. (d) Contour plot of $r_{3 \sigma}$ after $3.5 \mathrm{~h}$ of CHA amplification across a range of $\mathrm{H} 1$ and $\mathrm{H} 2$ concentrations, contours are smoothed between 32 experimentally determined values of $r_{3 \sigma}$ (Figure S1). (e, f) Scheme of predicted fluorescence intensities based on experimental data for positive $(+\mathrm{C} 1)$ and negative $(-\mathrm{C} 1)$ droplets for $[\mathrm{H} 1]=[\mathrm{H} 2]$ $=25 \mathrm{nM}$ and $[\mathrm{H} 1]=50 \mathrm{nM},[\mathrm{H} 2]=100 \mathrm{nM}$ respectively. $(\mathrm{g})$ Real-time signal amplification for catalyst $\mathrm{C} 5$ and five equivalents of catalyst $\mathrm{C} 1$ under optimised circuit conditions. 


\section{Optimisation of circuit composition}

During droplet encapsulation, the probability of a discrete number of molecules $(k)$ being contained within any particular droplet follows a Poisson distribution, where $\lambda$ is the expected number of molecules encapsulated for a given concentration (Equation 1). ${ }^{32}$

$$
P(k, \lambda)=\frac{\lambda^{k} e^{-\lambda}}{k !}
$$

In digital sensing applications, the expected number of analyte molecules per droplet is typically less than one, meaning that a majority of droplets contain no analyte. According to the ratio of droplets that contain the analyte $(k \geq 1)$ to those that do not $(k=0)$, the probability of encapsulation can be found, allowing calculation of $\lambda$ and thus the bulk analyte concentration.

In our case, for optimum assay performance we must also consider the Poisson loading of the $\mathrm{H} 1, \mathrm{H} 2$ and RQ CHA assay reagents as well as that of the analyte, due to the sub-picolitre volume of the droplets employed which results in significant variation in reagent concentrations between droplets (Figure 1(b)). In order to find optimal reagent concentrations for hairpins H1, H2 and the fluorescence signalling duplex RFQ, we consider the statistics of their droplet encapsulation. In digital droplet assays, it is typically only Poissonic encapsulation of the analyte that is considered and which is used to back-calculate the bulk analyte concentration from the proportion of positive and negative droplets. In our case, the Poisson loading of the CHA reagents is also relevant, due to the sub-picolitre volume of the droplets employed (Figure 1(b)). For example, the $\pm 3 \sigma$ range of actual droplet concentration from an encapsulated $25 \mathrm{nM}$ solution will vary between 23.2 and $26.8 \mathrm{nM}$, using the approximation that for sufficiently large $\lambda(\lambda>1000)$ the Poisson distribution is described by a normal distribution with mean equal to variance $\left(\mu=\lambda^{2}\right)$ according to Equation (2).

$$
P(k, \lambda>1000)=\frac{\lambda^{k} e^{-\lambda}}{k !} \cong \frac{1}{\sqrt{2 \pi \lambda^{2}}} e^{\frac{-(k-\lambda)^{2}}{2 \lambda^{2}}}
$$

Since the signal-to-noise ratio of the CHA circuit is affected by reagent concentration, this droplet-to-droplet variation in reaction conditions has a significant impact upon the combined signalto-noise ratio of positive and negative droplets, by broadening the distribution of fluorescence intensity in the positive and negative signals. For droplet identity to be accurately determined, we impose the condition that the mean fluorescence intensity of positive droplets must be greater than that of negative droplets with a confidence of at least $3 \sigma$ from both the positive and negative mean intensity (Equation 3).

$$
r_{3 \sigma}=\frac{I\left(\lambda_{\text {positive }}-3 \sigma_{\text {positive }}\right)}{I\left(\lambda_{\text {negative }}+3 \sigma_{\text {negative }}\right)}>1
$$

With [C1] $=15 \mathrm{pM}$, corresponding to the detection limit for CHA reaction, we conducted bulk-phase experiments to model $r_{3 \sigma}$ across a range of reagent concentrations in order to establish the feasibility of the ddCHA approach. Mean H1 and H2 concentrations were varied between 10-50 and 10-100 nM with $[\mathrm{RFQ}]=[\mathrm{H} 1]$. Variation in reagent concentration between $\lambda_{+C 1}-3 \sigma$ and $\lambda_{-C 1}+3 \sigma$ produced significant variation in the degree of CHA amplification and leakage $3.5 \mathrm{~h}$ after circuit initiation for the catalysed and non-catalysed reaction, respectively. For example, where $\lambda_{H 1}=\lambda_{H 2}=25 \mathrm{nM}, r_{\lambda}=$ 
$\frac{I\left(\lambda_{\text {positive })}\right.}{I\left(\lambda_{\text {negative })}\right.}=1.18$ whereas $r_{3 \sigma}=1.07$ (Figure $1(\mathrm{c})$ ). This experiment demonstrates the necessity to account for Poisson loading of the CHA reagents, as the observed variation in $r_{3 \sigma}$ with respect to $r_{\lambda}$ represents a major source of systematic noise in the resultant droplet assay.

Through systematic variation of $\mathrm{H} 1$ and $\mathrm{H} 2$ concentration, maximal values of $r_{3 \sigma}$ were found in the range $\lambda_{H 1}=25-30 \mathrm{nM}$ and $\lambda_{H 2}=25-40 \mathrm{nM}$ (Figure 1(d)). The maxima in $r_{3 \sigma}$ can be rationalised by considering the variation in signal strength resulting from circuit leakage and background versus the rate of catalysed reaction as a function of hairpin concentration. The sources of circuit leakage have been investigated previously,15 and are described further in the Supporting Information.

From the experimental fluorescence intensities for $\lambda, \lambda_{+C 1}-3 \sigma$ and $\lambda_{-C 1}+3 \sigma$ for catalysed and non-catalysed reactions, respectively, the distribution of positive and negative droplet fluorescence intensity can be predicted. Sufficient conditions for ddCHA exist where there is no overlap between the distribution of fluorescence intensities for the catalysed and non-catalysed reaction, as for $\lambda_{H 1}=$ $\lambda_{H 2}=25 \mathrm{nM}$ (Figure 1(e)), but not for $\lambda_{H 1}=50 \mathrm{nM}$ and $\lambda_{H 2}=100 \mathrm{nM}$, where $r_{3 \sigma}<1$ (Figure 1(f)). These data demonstrate that although higher hairpin concentrations $(>70 \mathrm{nM})$ result in greater signalling fluorescence, the concomitant increase in circuit leakage and background signal limits the signal:noise ratio. Thus, maximal $r_{3 \sigma}$ is found at lower hairpin concentrations such as $\sim 25 \mathrm{nM}$ as shown here, where the rate of catalysed reaction is fastest in comparison to leakage.

From these data, we believe that in the absence of additional sources of noise the CHA circuit can be an effective means for digital droplet sensing of catalyst $\mathrm{C} 1$. However, we envisioned that experimental noise, for example due to small variations in droplet volume and sampling error of droplet fluorescence, would broaden the recorded fluorescence distribution and reduce the effectiveness of ddCHA. To address this, we further improved the signal to noise ratio of the assay by increasing the turnover rate of the CHA reaction, by designing a CHA catalyst that contained five repeat units of the initial $\mathrm{C} 1$ sequence (C5, Figure 1(a)). Whilst a higher encapsulated concentration of catalyst could be achieved by decreasing the droplet size, this would also increase Poissonic variation in the concentration of the other CHA reagents and require higher throughput in the droplet assay. As expected, C5 was found to have a catalytic efficiency identical to five stoichiometric equivalents of C1 (Figure $1(\mathrm{~g})$ ), while experiments with catalyst $\mathrm{C} 5$ at various $\mathrm{H} 1$ and $\mathrm{H} 2$ concentrations showed $r_{3 \sigma}$ was maximised at similar conditions as for catalyst C1 (Figure S1).

Finally, we varied the concentration of RFQ to find $r_{3 \sigma}$ was optimised when [RFQ] $=10$ $\mathrm{nM}$. With these modifications, values $r_{\lambda}=3.2$ and $r_{3 \sigma}=1.42$ were found for the C5 catalyst with $[\mathrm{H} 1]=[\mathrm{H} 2]=25 \mathrm{nM}$ and $[\mathrm{RFQ}]=10 \mathrm{nM}$ (Figure $1(\mathrm{~g})$ ), conditions that we predicted would be suitable for effective ddCHA. 

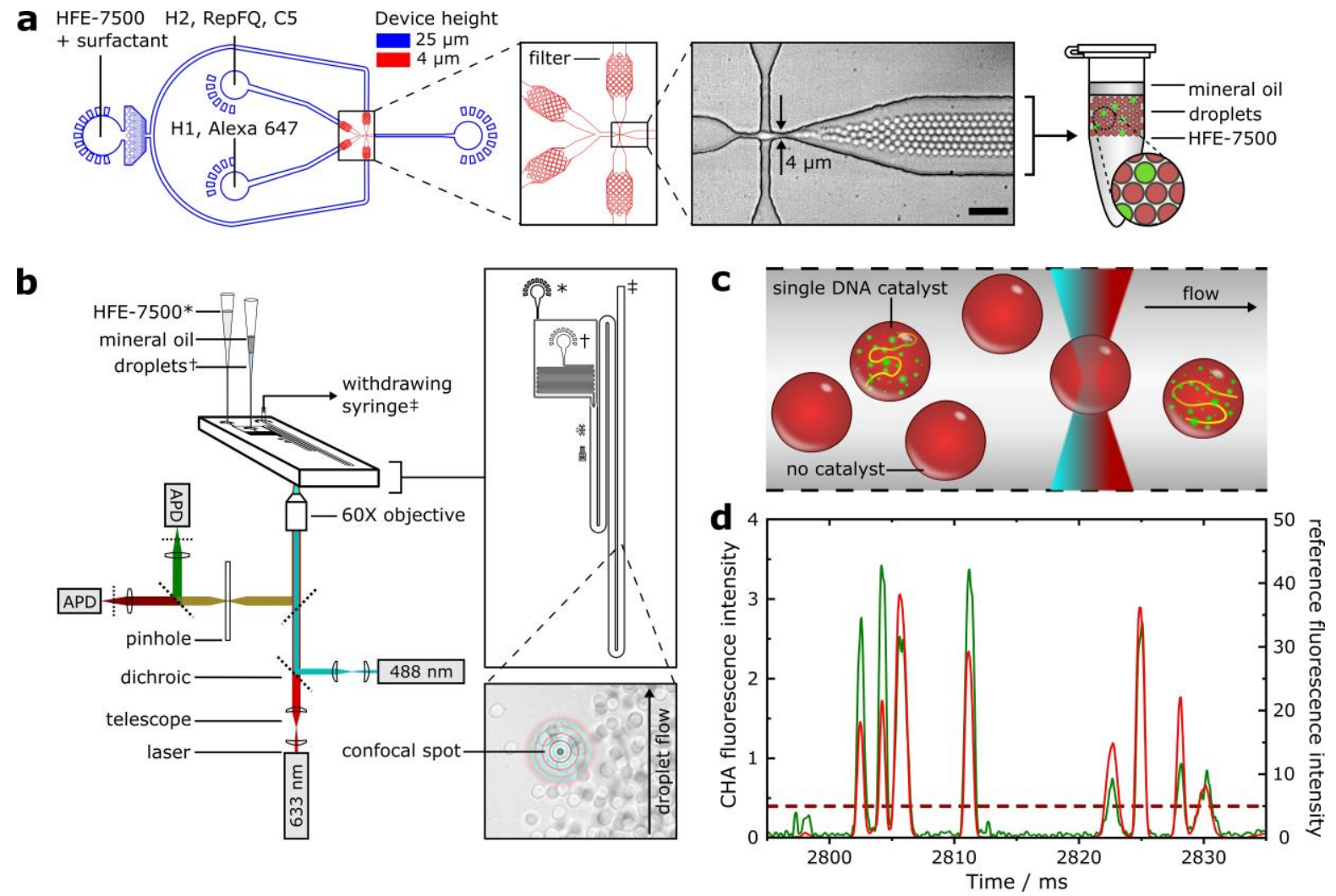

Figure 2: Microfluidic droplet generation, incubation and fluorescence assay. (a) Schematic of twolayer droplet-generating device, showing droplet formation through the $4 \times 4 \mu \mathrm{m}$ cross- section flowfocussing nozzle and subsequent incubation. (b) Schematic of optical apparatus and microfluidic chip for sequential two-colour confocal fluorescence assay of droplets. $*, \uparrow$ and $\ddagger$ denote HFE-7500, droplet and withdrawing syringe connections to the chip, respectively. (c) Cartoon of droplets passing through confocal volume at various heights within the device, producing the range of fluorescence intensities observed in (d). (d) Time trace of CHA signal and reference dye fluorescence for droplets passing sequentially through the two-colour confocal volume. Dashed line indicates threshold for minimum reference intensity.

\section{Droplet generation and fluorescence assay}

Following the optimisation of appropriate conditions for the DNA circuit, we designed a protocol for the generation and analysis of microdroplets for ddCHA sensing. We engineered a microfluidic droplet generator for the generation of droplets with diameter $\sim 6 \mu \mathrm{m}$ (volume $\sim 110 \mathrm{fL}$, Figure 2(a)). Under these conditions, single molecules of C5 are encapsulated at a concentration of $15 \mathrm{pM}$. From our analysis, we predict this to be sufficient for an sufficient signal:noise ratio between positive and negative droplets to be observed, whilst the droplet volume remains acceptably large to facilitate reasonable throughput of assay volume.

The droplet generator is of similar two-layer design to those employed elsewhere, ${ }^{5,33}$ with droplet formation at a $4 \times 4 \mu \mathrm{m}$ flow-focussing constriction at a rate of $\sim 20 \mathrm{kHz}$ (Video S1). H2, RFQ and $\mathrm{C} 5$ are introduced through a different inlet to $\mathrm{H} 1$ so that mixing and circuit initiation only occur after droplet encapsulation; droplets formed in this manner are highly monodisperse with a mean volume of $107 \pm 3.6 \mathrm{fL}$ (Figure S2). To enable single-droplet normalisation of CHA fluorescence 
intensity, a $10 \mu \mathrm{M}$ solution of poly- $\mathrm{T}_{21}$ oligo conjugated to a red-fluorescent dye is added alongside $\mathrm{H} 1$ as a reference for two-colour fluorescence assay of the droplets.

Following incubation, droplets are passed through a microfluidic chip in which the fluorescence signal of the CHA circuit and reference dye is recorded by two-colour coincidence detection (TCCD) microscopy (Figure 2(b, c)). Droplets and carrier oil are drawn through the microfluidic chip in 1:10 ratio, so that diffusion of the droplets can introduce inter-droplet spacing before passage through the confocal volume (Figure S3). The passage of droplets with simultaneous acquisition by two-colour confocal microscopy of both reference and CHA output fluorescence is observed as periodic bursts of fluorescence as a function of time (Figure 2(d)). The TCCD droplet assay is described further in the SI.

As the cross-sectional area of the flow chamber is larger than the droplets, droplets vary in their trajectory through the confocal volume, necessitating two-colour fluorescence assay in order to effectively normalise the CHA signal output on a droplet-by-droplet basis. An additional benefit of the two-colour approach is the normalisation of fluorescence intensity variations that result from fluctuations in microfluidic flow velocity. These occur in practice due to the parabolic flow profile present on chip and the exact placement of the confocal volume within it. ${ }^{34,35}$

Combined together, the two fluidic elements of droplet generation and analysis form an effective assay workflow. Droplets are generated in sufficient quantities within $\sim 20$ mins, while TCCD microscopy under microfluidic flow provides accurate quantitation of droplet fluorescence.

\section{Digital sensing of DNA input by CHA}

Having optimised the circuit conditions and following development of an effective and robust protocol for droplet generation, incubation and TCCD assay, we conducted experiments to demonstrate the feasibility and sensitivity of ddCHA (Figure 3).

At a nominal analyte concentration ([C5]) of $10 \mathrm{pM}$, corresponding to an average of 0.68 analyte molecules per droplet $\left(\lambda_{\text {prepared }}=0.68\right)$, discrete populations in a scatter plot of reference droplet fluorescence intensity $\left(I_{R}\right) v s$. CHA signalling intensity $\left(I_{C H A}\right)$ could be observed (Figure 3(a)). Plotting these data as a histogram of reference-normalised droplet CHA signalling intensity $\left(I_{C H A}^{N}=\right.$ $\left.\frac{I_{C H A}}{I_{R}}\right)$ resulted in distinct peaks, occurring at regular intervals as a function of $I_{C H A}^{N}$ (Figure 3(b)). In the absence of analyte only a single peak in $I_{C H A}^{N}$, corresponding to droplets weakly fluorescing from signal leakage alone, was observed (Figure 3(c)).

Each peak corresponds to a population of droplets with a discrete number of encapsulated analyte molecules, as verified by Gaussian peak fitting and integration which revealed peak integrals consistent with a Poisson distribution for $\lambda_{\text {reported }}=0.77$, corresponding to a reported $\mathrm{C} 5$ concentration of $11 \mathrm{pM}$ (Figure S5). In addition, the ratio between $I_{C H A}^{N}$ distribution maxima for droplets containing one and zero analyte molecules was equal to $\left.3.30 \frac{I_{C H A}^{N}(\mathrm{k}=1)}{I_{C H A}^{N}(\mathrm{k}=0)}=3.30\right)$, similar to that recorded in bulk-phase amplification experiments for [C5] $=15 v s$. [C5] $=0\left(\frac{I_{1 C 5}^{N}(\mathrm{t}=3.5)}{I_{0}^{N}(\mathrm{t}=3.5)}=3.32\right.$; Figure $1(\mathrm{~g}))$. Furthermore, the relative values of $\sigma$ for the Gaussian $I_{C H A}^{N}$ distributions for positive $(k>0)$ and negative $(k=0)$ droplets were in agreement with bulk experimental values for $I_{\lambda \pm 3 \sigma}$, with $\sigma(k=0)<\sigma(k>0)$. Together, this evidences the digital nature of the droplet assay, with the regular spacing between the Gaussian droplet intensity distributions consistent with $k=0,1,2,3 \ldots$ molecules of C5 per droplet. 

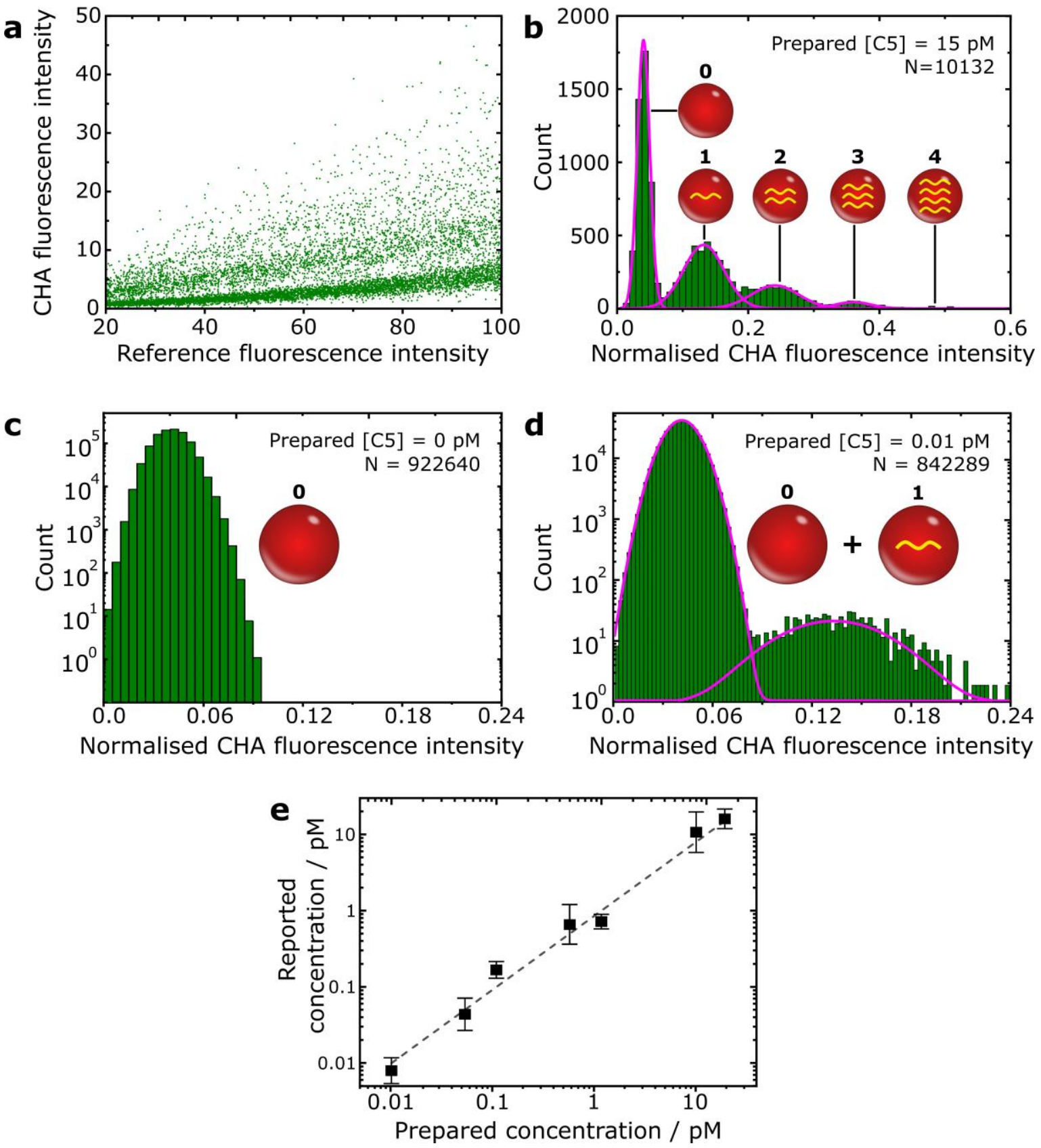

Figure 3: Experimental data from ddCHA experiments. (a) Thresholded scatter plot of sin- gle droplet reference vs. CHA signalling fluorescence intensity, example pre-threshold data is shown in the Supporting Information (Figure S3). (b) Histogram of normalised droplet CHA fluorescence intensity for droplets formed from $15 \mathrm{pM}$ bulk C5 concentration. Magenta lines are Gaussian fits to discrete histogram peaks. (c) Histogram and Gaussian fits of normalised droplet CHA fluorescence intensity for droplets formed from $10 \mathrm{fM}$ bulk C5 concentration. (d) Scatter plot of prepared vs. reported concentration of $\mathrm{C} 5$, as determined by ddCHA reaction. Error bars correspond to the standard deviation of three repeat measurements. Dashed line is least-squares fit with $\mathrm{r}^{2}=0.996,[\mathrm{H} 1]=[\mathrm{H} 2]=$ $25 \mathrm{nM},[\mathrm{RFQ}]=10 \mathrm{nM}$ in all experiments. 
After establishing the principle of digital sensing by ddCHA we conducted experiments to determine the detection limit. Due to its digital nature, this is dictated by the throughput of the droplet TCCD assay. Of the droplets recorded, approximately $39 \%$ are retained after thresholding and with an average droplet throughput of $202 \mathrm{~Hz}$, a $10 \mathrm{fM}$ concentration of C5 could be accurately reported within three hours (Figure 3(d)). This represents an increase in assay sensitivity of at least two orders of magnitude in comparison to the bulk reaction. Additional experiments at analyte concentrations ranging between $0.01-15 \mathrm{pM}$ were conducted to verify the sensing accuracy of ddCHA (Figure 3(e)). A linear fit in prepared analyte concentration against that reported by ddCHA was obtained, demonstrating accurate sensing over three orders of magnitude.

In an ideal digital assay, it is possible to binarise single signalling events into positive or negative bins with $100 \%$ certainty. However, despite optimisation of the CHA circuit conditions, overlap between $I_{C H A}^{N}$ distributions is observed for the ddCHA experiment $\left(r_{3 \sigma}^{d d C H A}<1\right)$ which introduces error into droplet binarisation. We attribute this distribution broadening to additional sources of noise such as sampling error in the TCCD assay and small variations in droplet volume. With Gaussian fitting of the $k=0$ and $k=1$ populations, the error in droplet assignment can be calculated as a function of bulk analyte concentration by considering the overlap integral of the two distributions (Supporting Information) as a function of analyte concentration. From this analysis, we ascertain that for analyte concentrations of $>70 \mathrm{fM}$ droplet binarisation can be performed with at least 95\% confidence. This indicates that the relatively small degree of overlap between the $k=0$ and $k=$ 1 distributions is not greatly detrimental to assay performance.

\section{Boolean logic operations by a digital DNA computer}

Having established and characterised DNA sensing by ddCHA, we then demonstrate a further application of ddCHA by employing it as a signal transducer for molecular computation. In DNAbased programming, strand displacement reactions function as Boolean logic gates, which can be combined to perform mathematical operations and function as neural networks. ${ }^{30,36}$ In most displacement-based circuits gate, outputs are communicated by fluorescence. In order to determine the positive (1) or negative (0) output of a gate, this signal must be accurately thresholded, requiring relatively high concentrations of gate nucleotides for reliably strong signal:noise ratio between 1 and 0 output. However, for truly digital molecular computation a single bit of information, in this case represented by a single DNA strand of specific sequence, should be addressable. To this aim, we have adapted the ddCHA reaction to function as a digital readout for simple Boolean operations.

Through selective inhibition of CHA, we engineered logic gates capable of AND or NOT operations (Figure 4(a)). The CHA mechanism is initiated by binding of the catalyst $1^{*}$ domain to the complementary sticky-end domain (1) in hairpin H1, introduction of catalyst inhibitor Ci therefore prevents $\mathrm{CHA}$ by masking the $1^{*}$ domain of the CHA catalyst. Addition of inhibitor complement $i^{*}$ returns full CHA activity, by sequestering $\mathrm{Ci}$ through strand displacement of $\mathrm{Ci}$ from $\mathrm{C} 5$. As $\mathrm{i}^{*}$ only contains part of the $1^{*}$ domain sequence, it does not itself catalyse or inhibit the rest of the CHA circuit (Figure 4(b)). Thus, when the CHA catalyst is inhibited as C5:(Ci)5, CHA reaction can only occur in the presence of both $\mathrm{C} 5:(\mathrm{Ci}) 5$ and $\mathrm{i}^{*}$ (AND gate), whilst uninhibited C5 will catalyse CHA except in the presence of $\mathrm{Ci}$ (NOT gate, Figure 4(c)). Furthermore, we propose that OR gates could be constructed by the simultaneous operation of orthogonal CHA circuits, where the presence of catalyst sequences for either of the circuits triggers a positive CHA signal. 


\section{a}
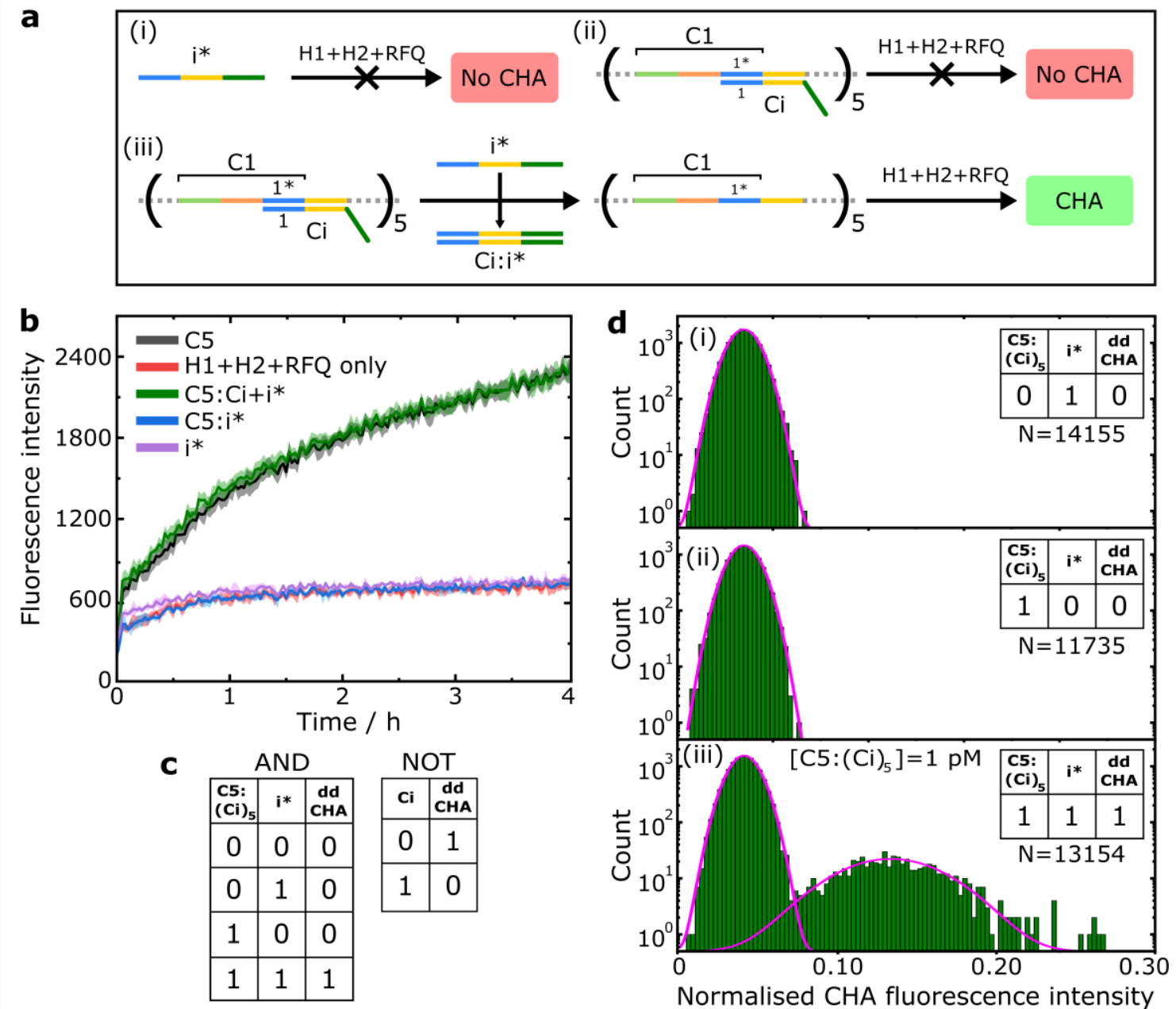

Figure 4: Digital logic computation by ddCHA. (a) Reaction schemes for gated CHA amplifi- cation. (i) Inhibitor complement strand $i^{*}$ lacks the necessary sequence to catalyse CHA. (ii) Catalyst inhibitor strand $\mathrm{Ci}$ binds to a catalyst sequence of $\mathrm{C} 5$, masking the $1^{*}$ domain required for circuit initiation via toehold-binding to domain 1 of hairpin H1. (iii) Inhibitor complement strand $\mathrm{i}^{*}$ displaces Ci from C5 via toehold binding and strand exchange, allowing uninhibited C5 to catalyse CHA. (b) Bulk-phase assay of CHA reaction with different gate inputs, only uninhibited C5 produces sufficient $\mathrm{CHA}$ amplification. Shaded error bars indicate standard deviation of three repeat measurements. (c) Truth tables for AND and NOT logic gates constructed by selective inhibition of CHA. (d) ddCHA data corresponding to operation of the AND logic gate. (i) $i^{*}$ input only. (ii) C5:(Ci) $)_{5}$ input only. (iii) $i^{*}$ and $\mathrm{C} 5:(\mathrm{Ci})_{5}$ inputs together, $\left[\mathrm{C} 5:(\mathrm{Ci})_{5}\right]=1 \mathrm{pM}$. $[\mathrm{H} 1]=[\mathrm{H} 2]=25 \mathrm{nM},[\mathrm{RFQ}]=10 \mathrm{nM}$ in all cases. 
ddCHA was then employed to apply the AND logic gate in a digital context (Figure 4(d)). ddCHA was selectively initiated only in the presence of both $\mathrm{C} 5:(\mathrm{Ci}) 5$ and $\mathrm{i}^{*}$, with $\mathrm{C} 5:(\mathrm{Ci}) 5$ and $\mathrm{i}^{*}$ alone not resulting in any positive ddCHA events, demonstrating AND computation at a singlemolecule level.

In solid-state processor design, the gate density, as afforded by their physical dimensions, is a fundamental aspect of computing performance. Analogously, gate density in DNA-based computing is given by the minimum number of identical DNA strands required to perform a logic function. In the data shown here, $\mathrm{C} 5:(\mathrm{Ci}) 5$ is present at $1 \mathrm{pM}$ concentration, representing a potential increase in gate density by a factor of 104 in comparison to conventional DNA circuits, where gate concentrations are typically on the order of tens of nanomolar. Although careful engineering of existing circuit designs may produce gates capable of sensing sub-nano molar signals, it is likely that this would come at the cost of greatly reduced signal-to-noise and computational speed. Robust operation of the gates required $\mathrm{Ci}$ and $\mathrm{i}^{*}$ concentrations of $10 \mathrm{nM}$ due to the relatively weak binding equilibrium between them and C5 present at pM concentration. However, it is feasible that computational circuits could be designed to utilise longer DNA oligos, with subsequently stronger hybridisation equilibria. This would enable the operation of logic circuits with all components present at $\mathrm{pM}$ or sub-pM concentrations. Crucially, however, this work demonstrates that single-molecule output from logic circuits can be transduced by ddCHA, enabling digital molecular computation. Moreover, the isothermal and enzymefree manner of ddCHA is entirely compatible with DNA-computing protocols, whilst the necessity for signal amplification by the gate output makes signal transduction highly specific and resistant to experimental noise.

\section{Conclusions}

We have engineered a digital droplet assay to achieve single-molecule sensitivity in signal amplification by catalysed hairpin assembly, using commercially available materials and without additional reagent purification. This approach effectively eliminates conventional challenges in isothermal DNA circuitry associated with off-pathway signal leakage. We have demonstrated the application of ddCHA to molecular computing, and shown that ddCHA has the potential to significantly enhance the sensitivity and processing density of DNA circuits. More broadly, ddCHA has a wide range of possible bioanalytical applications, with the sensitivity limits demonstrated here approaching those of conventional techniques such as PCR. The microfluidic workflow, enzyme-free and isothermal nature of CHA makes it highly appropriate for point of care applications. ${ }^{37}$ Furthermore, CHA can be adapted to sense substrates other than DNA, with RNA and protein biomarkers potential targets for future ddCHA-based assays. ${ }^{38,39}$

\section{Methods}

\section{Solutions and reagents}

All oligonucleotides were purchased HPLC-purified from Integrated DNA Technologies (Coralville, USA). Oligonucleotide sequences are summarised in the Supplementary Table 1. Stock solutions of oligonucleotides, diluted to $100 \mu \mathrm{M}$, were stored in TE buffer ( $\mathrm{pH}$ 7.5). Lower concentration oligonucleotide stocks were diluted in TNaK buffer (20 mM Tris, $\mathrm{pH} 7.5 ; 140 \mathrm{mM} \mathrm{NaCl} ; 5 \mathrm{mM} \mathrm{KCl})$, stocks of concentration $<1 \mu \mathrm{M}$ were supplemented with $1 \mu \mathrm{M}$ poly- $\mathrm{T}_{21}$ to reduce surface adsorption. All oligonucleotides were purchased HPLC-purified from Integrated DNA Technologies (Coralville, USA). 
Oligonucleotide sequences are summarised in the Supplementary Table 1. Stock solutions of oligonucleotides, diluted to $100 \mathrm{mM}$, were stored in TE buffer ( $\mathrm{pH}$ 7.5). Lower concentration oligonucleotide stocks were diluted in TNaK buffer (20 mM Tris, pH 7.5; $140 \mathrm{mM} \mathrm{NaCl} ; 5 \mathrm{mM} \mathrm{KCl}$ ), stocks of concentration $<1 \mathrm{mM}$ were supplemented with $1 \mathrm{mM}$ poly-T21 to reduce surface adsorption. Prior to use, DNA hairpins, duplex RFQ (RF and RQ mixed in 1:2 molar ratio) and gate component C5:(Ci)5 (C5 and Ci mixed in 1:10 molar ratio) were annealed in $\mathrm{TNaK}$ buffer by heating to $90{ }^{\circ} \mathrm{C}$, followed by cooling at $0.1{ }^{\circ} \mathrm{C} / \mathrm{s}$ to ambient temperature. Kinetic experiments were conducted at $37{ }^{\circ} \mathrm{C}$ using a plate reader (96-well half-area, CLARIOstar, BMG Labtech).

\section{Bulk kinetic assays}

Kinetic experiments were conducted at $37{ }^{\circ} \mathrm{C}$ using a plate reader (96-well half-area, CLARIOstar, BMG Labtech). Reaction mixtures in TNaK buffer supplemented with $1 \mu \mathrm{M}$ poly-T21 were plated to a total well volume of $100 \mu \mathrm{L}$, and pre-warmed before circuit initiation by the addition of H1. Logic gate operation in bulk solution were conducted analogously, with $\mathrm{C} 5, \mathrm{Ci}$ and i* concentrations of $15 \mathrm{pM}$, $10 \mathrm{nM}$ and $10 \mathrm{nM}$, respectively.

\section{Microfluidic device fabrication}

Devices were designed using AutoCAD software (Autodesk) and photolithographic masks printed on acetate transparencies (Micro Lithography Services, Chelmsford, UK). Polydimethylsiloxane (PDMS) devices were produced on SU-8 moulds fabricated via photolithographic processes as described elsewhere, ${ }^{40}$ with UV exposure performed with a custom-built LED-based apparatus. ${ }^{41}$ Multilayer moulds were fabricated by sequential photolithographic steps, alignment between the lithography processes was achieved using a custom-built mask aligner including a rotating xyz stage (ThorLabs, MBT602/M and PR01/M). Further details are given in the Supporting Information.

\section{ddCHA reagent preparation}

Separate DNA solutions containing appropriate concentrations of C5, H2 and RFQ (Solution A) and H1 (Solution B) were mixed with $400 \mathrm{nM}$ Alexa647-pT21 conjugated DNA before microfluidic droplet emulsification. In the case of logic-gated ddCHA, gate components C5 (1 pM), Ci (10 nM) and i* (10 $\mathrm{nM}$ ) were incubated together with CHA circuit components in Solution A at $37^{\circ} \mathrm{C}$ for thirty minutes prior to droplet generation with simultaneous mixing of Solution B. Gate components $\mathrm{Ci}$ and $\mathrm{i}^{*}$ were added at $10 \mathrm{nM}$ concentration relative to $\mathrm{C} 5$.

\section{Droplet generation and incubation}

Pre-filtered solutions of oligonucleotides and HFE-7500 oil supplemented with $1.5 \% \mathrm{w} / \mathrm{v}$ triblockcopolymer fluorosurfactant (008, RAN biotechnologies) were introduced to the device through equal lengths of PTFE tubing (06417-11, Cole-Parmer) using an Elveflow OB1-MK3 pressure- driven flow controller equipped with three 0-2 bar channels (Elvesys, Paris, France). Aqueous solutions A and B were driven under 1 bar pressure, with the oil phase driven with 0.9 bar pressure for respective flow rates of 9 (per aqueous inlet) and $110 \mu \mathrm{L} / \mathrm{h}$. After allowing droplet generation to equilibrate ( $3 \mathrm{~min}$ ), droplets were collected continuously over 5-20 min using a gel-loading tip partially prefilled with mineral oil (Sigma Aldrich) to prevent droplet evapora- tion, inserted into the device outlet. Droplets were then transferred into an Eppendorf tube and incubated under mineral oil at $37{ }^{\circ} \mathrm{C}$ for $3.5 \mathrm{~h}$ before fluorescence assay. To ensure droplet ho- mogeneity and accurate calculation of analyte concentration, an aliquot of droplets was retained and their size measured using optical microscopy for each 
experiment.

\section{Droplet fluorescence assay}

Devices were pre-filled with filtered HFE-7500, gel-loading tips containing filtered HFE-7500 and droplets under mineral oil were inserted into their respective inlets. Droplets and sheath-flow oil were drawn through the device at $150 \mu \mathrm{L} / \mathrm{h}$ by a syringe ( $1 \mathrm{~mL}$, Gastight, Hamilton) and syringe pump (PHD 2000, Harvard Apparatus) in withdrawing mode. A ratio in fluidic resistance of 1:10 between oil and droplet inlet channels determined a 10:1 relative flow-rate, promoting dispersion of the concentrated droplet mixture into the co-flowing oil.

\section{Confocal microscopy for coincident fluorescence detection}

Experiments were performed on a two-colour coincidence detection confocal microscope described previously ${ }^{42}$ details are provided in the Supplementary Information. The confocal spot was directed into the microfluidic droplet flow chamber, red and green-fluorescence traces resulting from droplet passage through the confocal volume were recorded, smoothed, thresholded (FigureS4) and processed by a Python script to afford peak intensities coincident between the red and green fluorescence channels.

\section{Supporting Information}

Supporting Information Available: additional experimental data and further descriptions of the methods employed in this article including information regarding circuit leakage in CHA reactions, droplet homogeneity, details of the TCCD assay, ddCHA data thresholding, error analysis in ddCHA, further experimental information and the nucleic acid sequences employed are avail- able free of charge via the Internet at http://pubs.acs.org.

\section{Acknowledgements}

The research leading to these results has received funding from the European Research Coun- cil under the European Union's Seventh Framework Programme (FP7/2007-2013) through the ERC grant PhysProt (agreement 337969) and from the Newman foundation. U.F.K was sup- ported by an ERC Consolidator grant (Designer-Pores 647144). W. E. A. acknowledges support from the EPSRC Cambridge NanoDTC, EP/L015978/1. Q. P. acknowledges funding by the European Union's Horizon 2020 research and innovation programme under ETN grant 674979- NANOTRANS. This work was supported by the Centre for Misfolding Diseases.

\section{References}

[1] Hynes, N. E.; Ingham, P. W.; Lim, W. A.; Marshall, C. J.; Massagué, J.; Pawson, T. Signalling Change: Signal Transduction through the Decades. Nat. Rev. Mol. Cell Biol. 2013, 14, 393-398.

[2] Lagnado, L. Signal Amplification: Let's Turn down the Lights. Curr. Biol. 2002, 12, 215- 217.

[3] Stoeger, T.; Battich, N.; Pelkmans, L. Passive Noise Filtering by Cellular Compartmentalization. Cell 2016, 164, 1151-1161.

[4] Murakoshi, H.; Iino, R.; Kobayashi, T.; Fujiwara, T.; Ohshima, C.; Yoshimura, A.; Kusumi, A. Single-Molecule Imaging Analysis of Ras Activation in Living Cells. PNAS 2004, 101, 7317- 
[5] Shim, J.; Ranasinghe, R. T.; Smith, C. A.; Ibrahim, S. M.; Hollfelder, F.; Huck, W. T. S.; Klenerman, D.; Abell, C. Ultrarapid Generation of Femtoliter Microfluidic Droplets for SingleMolecule-Counting Immunoassays. ACS Nano 2013, 7, 5955-5964.

[6] Choi, J.; Love, K. R.; Gong, Y.; Gierahn, T. M. Immuno-Hybridization Chain Reaction Enhances Detection of Individual Cytokine-Secreting Human Peripheral Mononuclear Cells. Anal. Chem. 2011, 83, 6890-6895.

[7] Jung, C.; Ellington, A. D. Diagnostic Applications of Nucleic Acid Circuits. Acc. Chem. Res. 2014, 47, 1825-1835.

[8] Liang, C.-P.; Ma, P.-Q.; Liu, H.; Guo, X.; Yin, B.-C.; Ye, B.-C. Rational Engineering of a Dynamic, Entropy-Driven DNA Nanomachine for Intracellular MicroRNA Imaging. Angew. Chem. Int. Ed 2017, 56, 9077-9081.

[9] Arter, W. E.; Charmet, J.; Kong, J.; Saar, K. L.; Herling, T. W.; Müller, T.; Keyser, U. F.; Knowles, T. P. Combining Affinity Selection and Specific Ion Mobility for Microchip Protein Sensing. Anal. Chem. 2018, 90, 10302-10310.

[10] Choi, H. M. T.; Schwarzkopf, M.; Fornace, M. E.; Acharya, A.; Artavanis, G.; Stegmaier, J.; Cunha, A.; Pierce, N. A. Third-Generation In Situ Hybridization Chain Reaction: Multiplexed, Quantitative, Sensitive, Versatile, Robust. Development 2018, 145.

[11] Chatterjee, G.; Dalchau, N.; Muscat, R. A.; Phillips, A.; Seelig, G. A Spatially Localized Architecture for Fast and Modular DNA Computing. Nat. Nanotechnol. 2017, 12, 920-927.

[12] Yin, P.; Choi, H. M. T.; Calvert, C. R.; Pierce, N. A. Programming Biomolecular Self-Assembly Pathways. Nature 2008, 451, 318-322.

[13] Dirks, R. M.; Pierce, N. A. Triggered Amplification by Hybridization Chain Reaction. PNAS 2004, 101, 15275-15278.

[14] Zhang, D. Y.; Turberfield, A. J.; Yurke, B.; Winfree, E. Engineering Entropy-Driven Reactions and Networks Catalyzed by DNA. Science (80-.). 2007, 318, 1121-1125.

[15] Chen, X.; Briggs, N.; McLain, J. R.; Ellington, A. D. Stacking Nonenzymatic Circuits for High Signal Gain. PNAS 2013, 110, 5386-91.

[16] Lv, Y.; Cui, L.; Peng, R.; Zhao, Z.; Qiu, L.; Chen, H.; Jin, C.; Zhang, X.-B.; Tan, W. Entropy Beacon: A Hairpin-Free DNA Amplification Strategy for Efficient Detection of Nucleic Acids. Anal. Chem. 2015, 87, 11714-11720.

[17] Li, B.; Ellington, A. D.; Chen, X. Rational, Modular Adaptation of Enzyme-Free DNA Circuits to Multiple Detection Methods. Nucleic Acids Res. 2011, 39, e110.

[18] Ottesen, E. A.; Hong, J. W.; Quake, S. R.; Leadbetter, J. R. Microfluidic Digital PCR Enables Multigene Analysis of Individual Environmental Bacteria. Science (80-.). 2006, 1464 1467. 
[19] Taylor, S. C.; Laperriere, G.; Germain, H. Droplet Digital PCR versus qPCR for Gene Expression Analysis with Low Abundant Targets: From Variable Nonsense to Publication Quality Data. Sci. Rep. 2017, 7, 2409.

[20] Benenson, Y.; Gil, B.; Ben-Dor, U.; Adar, R.; Shapiro, E. An Autonomous Molecular Computer for Logical Control of Gene Expression. Nature 2004, 429, 423-429.

[21] Zhang, D. Y.; Seelig, G. Dynamic DNA Nanotechnology Using Strand-Displacement Reactions. Nat. Chem. 2011, 3, 103-113.

[22] Woods, D.; Doty, D.; Myhrvold, C.; Hui, J.; Zhou, F.; Yin, P.; Winfree, E. Diverse and Robust Molecular Algorithms Using Reprogrammable DNA Self-Assembly. Nature 2019, 567, 366-372.

[23] Seelig, G.; Soloveichik, D.; Zhang, D. Y.; Winfree, E. Enzyme-Free Nucleic Acid Logic Circuits. Science (80-. ). 2006, 314, 1585-1588.

[24] Wu, C.; Wan, S.; Hou, W.; Zhang, L.; Xu, J.; Cui, C.; Wang, Y.; Hu, J.; Tan, W. A Survey of Advancements in Nucleic Acid-Based Logic Gates and Computing for Applications in Biotechnology and Biomedicine. Chem. Commun. 2015, 51, 3723-34.

[25] Yang, J.; Wu, R.; Li, Y.; Wang, Z.; Pan, L.; Zhang, Q.; Lu, Z.; Zhang, C. Entropy-Driven DNA Logic Circuits Regulated by DNAzyme. Nucleic Acids Res. 2018, 46, 8532-8541.

[26] Elbaz, J.; Yin, P.; Voigt, C. A. Genetic Encoding of DNA Nanostructures and Their SelfAssembly in Living Bacteria. Nat. Commun. 2016, 7, 11179.

[27] Groves, B.; Chen, Y.-J.; Zurla, C.; Pochekailov, S.; Kirschman, J. L.; Santangelo, P. J.; Seelig, G. Computing in Mammalian Cells with Nucleic Acid Strand Exchange. Nat. Nanotechnol. 2016, 11, 287-294.

[28] Wu, Z.; Fan, H.; Satyavolu, N. S. R.; Wang, W.; Lake, R.; Jiang, J.-H.; Lu, Y. Imaging Endogenous Metal Ions in Living Cells Using a DNAzyme-Catalytic Hairpin Assembly Probe. Angew. Chem. Int. Ed 2017, 56, 8721-8725.

[29] Li, D.; Wu, Y.; Gan, C.; Yuan, R.; Xiang, Y. Bio-Cleavable Nanoprobes for Target-Triggered Catalytic Hairpin Assembly Amplification Detection of MicroRNAs in Live Cancer Cells. Nanoscale 2018, 10, 17623-17628.

[30] Qian, L.; Winfree, E. Scaling Up Digital Circuit Computation with DNA Strand Displacement Cascades. Science (80-. ). 2011, 99, 1196-1202.

[31] Wilhelm, D.; Bruck, J.; Qian, L. Probabilistic Switching Circuits in DNA. PNAS 2018, 115, 903-908.

[32] Collins, D. J.; Neild, A.; DeMello, A.; Liu, A.-Q.; Ai, Y. The Poisson Distribution and Beyond: Methods for Microfluidic Droplet Production and Single Cell Encapsulation. Lab Chip 2015, 15, 3439-3459.

[33] Weinmeister, R.; Freeman, E.; Eperon, I. C.; Stuart, A. M.; Hudson, A. J. Single-Fluorophore Detection in Femtoliter Droplets Generated by Flow Focusing. ACS Nano 2015, 9, 9718-9730.

[34] Squires, T. M.; Quake, S. R. Microfluidics: Fluid Physics at the Nanoliter Scale. Rev. Mod. Phys. 
2005, 77, 977-1026.

[35] Horrocks, M. H.; Li, H.; Shim, J.-u.; Ranasinghe, R. T.; Clarke, R. W.; Huck, W. T. S.; Abell, C.; Klenerman, D. Single Molecule Fluorescence under Conditions of Fast Flow. Anal. Chem. 2012, 84, $179-185$.

[36] Qian, L.; Winfree, E.; Bruck, J. Neural Network Computation with DNA Strand Displacement Cascades. Nature 2011, 475, 368-372.

[37] Pandey, C. M.; Augustine, S.; Kumar, S.; Kumar, S.; Nara, S.; Srivastava, S.; Malhotra, B. D. Microfluidics Based Point-of-Care Diagnostics. Biotechnol. J. 2018, 13, 1-11.

[38] Bhadra, S.; Ellington, A. D. Design and Application of Cotranscriptional Non-Enzymatic RNA Circuits and Signal Transducers. Nucleic Acids Res. 2014, 42, e58-e58.

[39] Tang, Y.; Lin, Y.; Yang, X.; Wang, Z.; Le, X. C.; Li, F. Universal Strategy to Engineer Catalytic DNA Hairpin Assemblies for Protein Analysis. Anal. Chem. 2015, 87, 8063-8066.

[40] Mazutis, L.; Gilbert, J.; Ung, W. L.; Weitz, D. A.; Griffiths, A. D.; Heyman, J. A. Single-Cell Analysis and Sorting Using Droplet-Based Microfluidics. Nat. Protoc. 2013, 8, 870-891.

[41] Challa, P. K.; Kartanas, T.; Charmet, J.; Knowles, T. P. J. Microfluidic Devices Fabricated Using Fast Wafer-Scale LED-Lithography Patterning. Biomicrofluidics 2017, 11, 014113.

[42] Li, H.; Ying, L.; Green, J. J.; Balasubramanian, S.; Klenerman, D. Ultrasensitive Coincidence Fluorescence Detection of Single DNA Molecules. Anal. Biochem. 2003, 75, 1664- 1670. 